\title{
Contrast Sensitivity and Pattern ERG in Waldenstrom's Macroglobulinaemia
}

\author{
M. P. CLARKE, ${ }^{*}$ M. YAP, $†$ and J. R. WEATHERILL* \\ Bradford
}

\begin{abstract}
Summary
Patients with Waldenstrom's macroglobulinaemia (WM) and age matched controls have been examined using tests of contrast sensitivity and pattern electroretinography. The patients with WM were in remission on maintenance treatment and had only moderately elevated plasma viscosity. They showed evidence of abnormal contrast sensitivity but there was no significant difference in amplitude of the pattern ERG between patients and controls.
\end{abstract}

Waldenstrom's macroglobulinaemia (WM) is a disorder in which an abnormally high molecular weight serum paraprotein causes increased blood viscosity. ${ }^{1}$ This leads to disturbances of blood flow which can manifest as neurological and visual symptoms. ${ }^{2}$ Fundal examination shows irregularly dilated retinal veins, haemorrhages and microaneurysms. ${ }^{3}$ The retinal appearances and fluorescein angiogram in such a patient are shown in Figs. 1 and 2 (patient A.B.).

Removal of the paraprotein by plasmapheresis lowers the serum viscosity and may cause regression of the retinal abnormalities. ${ }^{4}$ This is illustrated in Fig. 3 of the same patient (A.B.) following plasmapheresis. A proportion of patients suffer irreversible visual loss. ${ }^{5}$

A group of patients undergoing treatment for WM and having only moderately raised serum viscosities have been investigated for the prescence of subtle abnormalities of retinal function using spatial and temporal con-

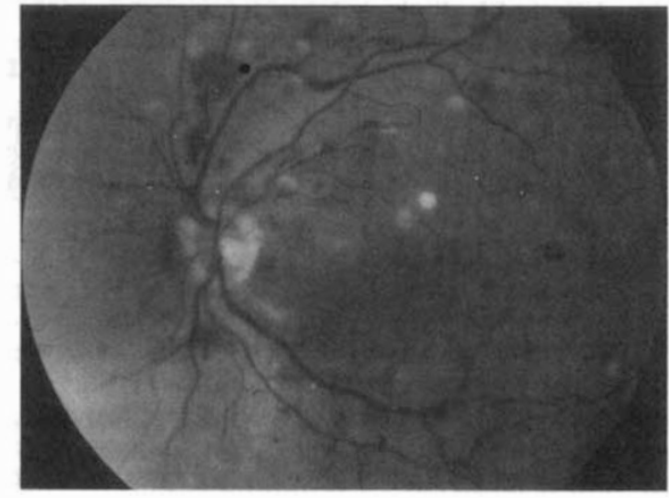

Fig. 1. Patient A.B. left fundus on presentation. Plasma viscosity 8 centipoise.

trast sensitivity techniques. The results have been compared with those of age matched controls. An attempt has been made to correlate these findings with the results of pattern electroretinography (PERG) performed on the same groups.

From Department of Ophthalmology, ${ }^{*}$ Bradford Royal Infirmary and the School of Optometry, Bradford University $\dagger$

Correspondence to Michael Clarke, FRCS, Department of Ophthalmology, Leeds General Infirmary, Great George St, Leeds LS1 3EX.

Presented at the Annual Congress of the Ophthalmological Society of the United Kingdom, April 1987. 


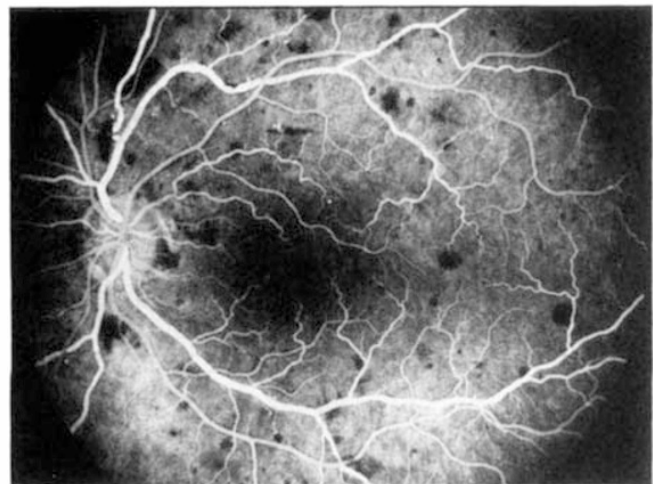

Fig. 2. Fluorescein angiogram of patient $A . B$.

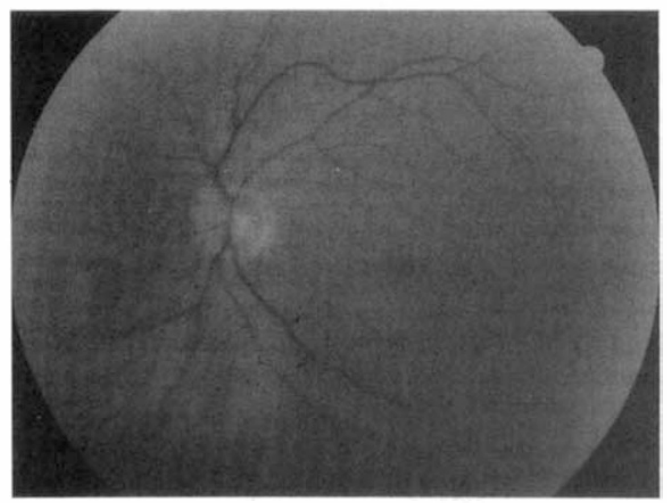

Fig. 3. Patient A.B. following plasmapheresis.

\section{Method}

Spatial and temporal contrast sensitivity measurements were recorded using the eye with the best visual acuity in each patient with WM. These results were compared with healthy age matched controls. The Snellen acuity was $6 / 6$ or better in three patients and 6/9 in one (D.B.). The media were clear in all cases.

Spatial contrast sensitivity was measured using an electronic monitor based system. The stimulus field covered $10 \times 7$ degrees and had a mean luminance of $50 \mathrm{~cd} / \mathrm{m}^{2}$. The surround luminance was less than $1 \mathrm{~cd} / \mathrm{m}^{2}$. Six spatial frequencies ranging from 0.5 to $16 \mathrm{cpd}$ were examined. The method of increasing contrast was used and threshold was taken as the mean of 5 readings for each spatial frequency.

Temporal contrast sensitivity was measured using an LED stimulus which subtended a visual angle of 2 degrees. The luminance of the LED was $130 \mathrm{~cd} / \mathrm{m}^{2}$ and the immediate surround was matched for luminance and colour. Five temporal frequencies ranging from 1.5 to $24 \mathrm{cps}$ were measured using the method of increasing contrast. Threshold was taken as the mean of 5 readings for each temporal frequency.

Pattern electroretinography (PERG) was undertaken using the DTL microfibre electrode ${ }^{6}$ inserted behind the lower lid and run from the lateral to medial canthus. Silver chloride coated electrodes were attached with electroconductive paste to the skin of the ipsilateral temple (reference) and to the forehead in the midline (ground). The inter-electrode impedance was less than 5 kilo-ohms. The stimulus was a black and white checkerboard generated by a Nicolet $100 \mathrm{G}$ visual stimulator reversing at $3 \mathrm{~Hz}$. The field size was $11.5 \times 8.5$ degrees at a viewing distance of 2.6 metres. Each check, subtended a visual angle of 30 minutes. The mean screen luminance was $158 \mathrm{~cd} / \mathrm{m}^{2}$ and the contrast of the checks was 80 per cent. The PERG was amplified using a Nicolet HEA200A amplifier connected to a Nicolet CA1000 signal averager and a DC2000 disc controller for data storage and analysis. Three hundred and sixty repetitions were recorded over $300 \mathrm{~ms}$ epochs and averaged in each case. The artefact rejection system was active during recording. Uniocular stimulation was used and recordings were taken from the eye on which contrast sensitivity measurements had been made earlier in the same session. The fellow eye was patched.

All psychophysical and electrophysiological tests were performed with the patient wearing their optimum spectacle correction appropriate for the test distance. No attempt was made to influence the natural pupil size.

The plasma viscosity was measured in all patients with WM. The ages of the patients with WM ranged from 64 to 82 years (mean 70 years) and the controls were 60 to 72 years (mean 68 years).

\section{Results}

The mean temporal and spatial contrast sensitivity functions for patients with $\mathrm{WM}$ and controls are displayed in Figs. 4a and 5a respectively. Findings for individual patients are shown in Figs. $4 \mathrm{~b}$ and $5 \mathrm{~b}$. These demonstrate temporal contrast sensitivity losses in all, and spatial contrast sensitivity losses in some patients with WM.

The PERG and plasma viscosity results are shown in Table $\mathrm{I}$. The amplitude of the response was calculated from the initial positive peak at 60 milliseconds to the subsequent trough at $90 \mathrm{~ms}$. There is a trend towards lower PERG amplitudes in the patients with WM compared with controls. This trend is not 


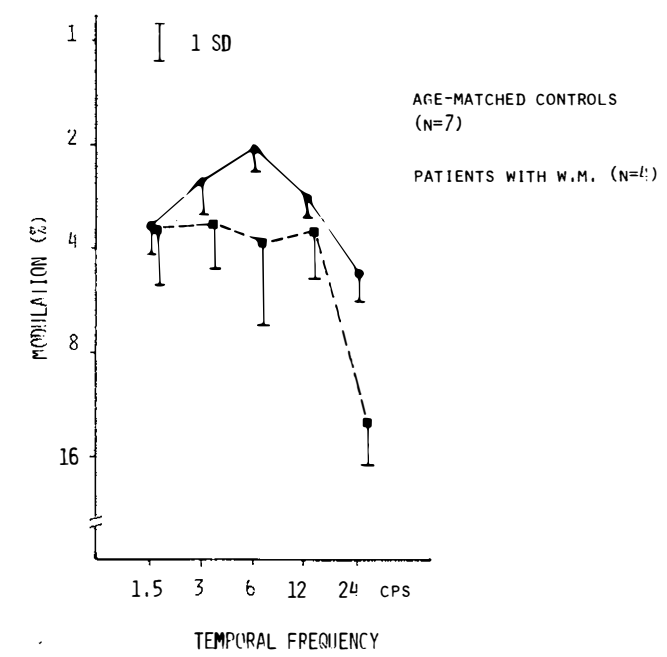

Fig. 4a. Mean temporal contrast sensitivity for patients with Waldenstrom's macroglobulinaemia, broken line: and controls, continuous line.

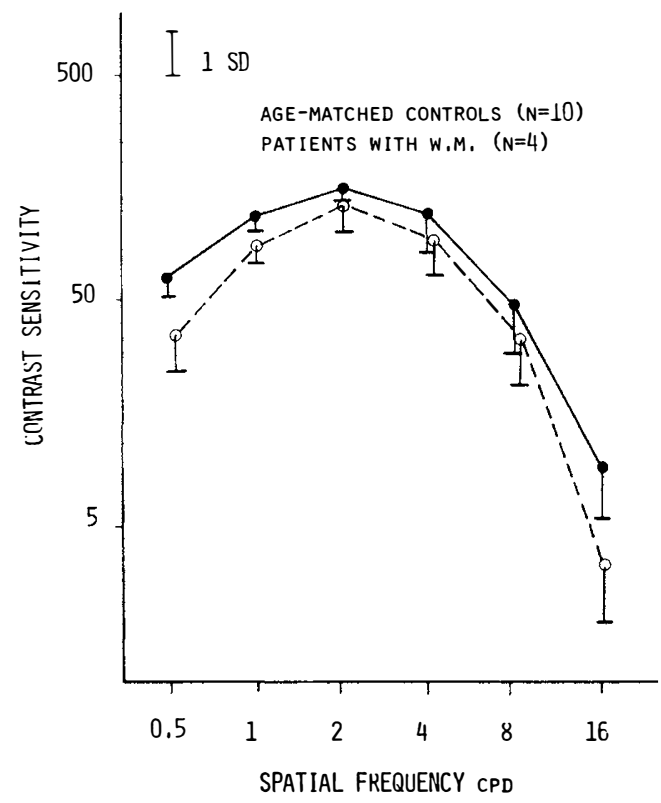

Fig. 5a. Mean spatial contrast sensitivity for patients with Waldenstrom's macroglobulinaemia, broken line: and controls, continuous line.

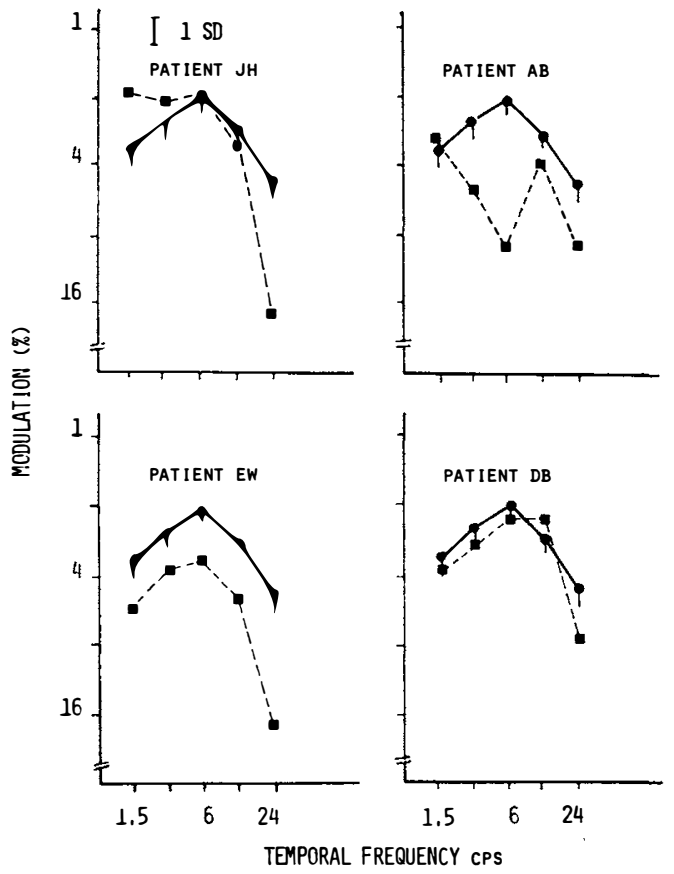

Fig. 4b. Temporal contrast sensitivity for individual patients with Waldenstrom's macroglobulinaemia, broken line: and normal values, continuous line.

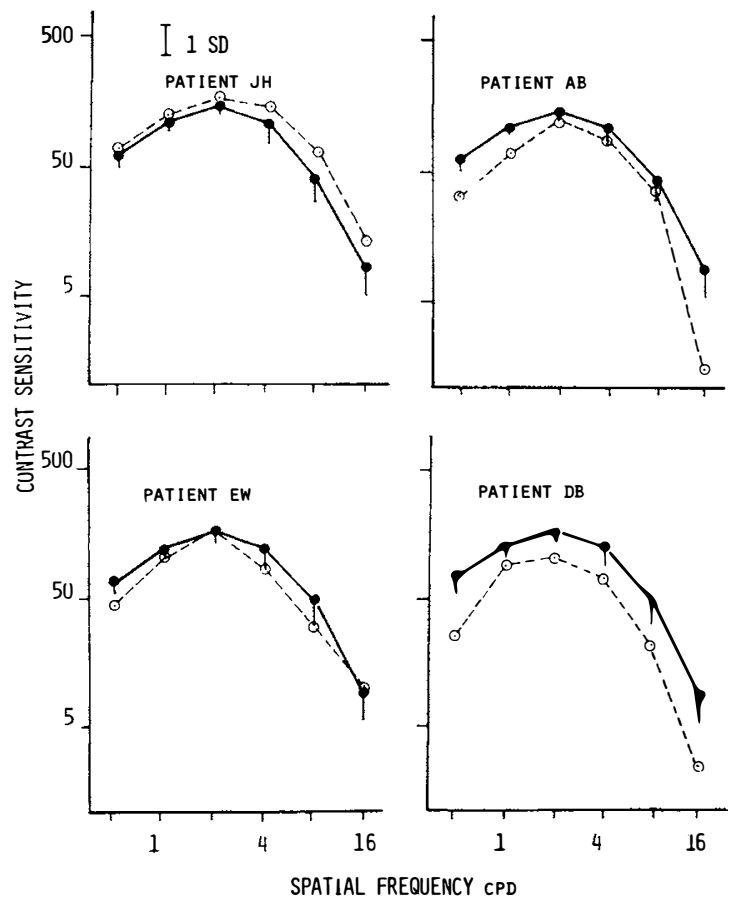

Fig. 5b. Spatial contrast sensitivity for individual patients with Waldenstrom's macroglobulinaemia, broken line: and normal vlaues, continuous line. 
Table I Pattern ERG data

\begin{tabular}{rcc}
\hline Patient No. & $\begin{array}{c}\text { Perg amplitude } \\
\text { (microvolts) }\end{array}$ & $\begin{array}{c}\text { Plasma viscosity } \\
\text { (centipoise) }\end{array}$ \\
\hline W.M.1 & 1.32 & 2.4 \\
2 & 1.2 & 2.2 \\
3 & 1.2 & 1.79 \\
4 & 2.75 & 2.2 \\
5 & 2.2 & 1.96 \\
6 & 2.65 & 1.87 \\
& & \\
Control 1 & 2.4 & \\
2 & 2.2 & \\
3 & 2.5 & Normal range \\
4 & 4.75 & $1.5-1.72$ cps \\
5 & 1.2 & \\
6 & 2.5 & \\
\hline
\end{tabular}

statistically significant and does not seem to relate to the plasma viscosity. It should be noted that at the time they were studied, the patients with WM were all undergoing treatment with chlorambucil or prednisolone or a combination of these two drugs. Some had previously undergone plasmapheresis in order to reduce viscosity levels up to 8 centipoise.

\section{Discussion}

Severe visual loss has been reported in WM and may result from hyperviscosity ${ }^{2}$ or from deposition of IgM at the macula. ${ }^{7}$ The reversal of hyperviscosity retinopathy by plasmapheresis which removes the abnormal paraprotein was first reported by Schwab et al. in $1960 .{ }^{4}$ This therapy does not always lead to visual recovery. ${ }^{5}$

There is less information available concerning the eyesight of patients who have less florid hyperviscosity. The hyperviscosity syndrome can affect patients suffering from leukaemia, polycythaemia and multiple myeloma, in addition to WM. ${ }^{8}$

It is well known that contrast sensitivity provides a more accurate measurement of visual function than Snellen acuity. ${ }^{9,10}$ The patients in this study showed abnormalities of contrast sensitivity despite normal Snellen acuity.

The PERG is thought to originate in the ganglion cell layer and has been shown to be abnormal in optic neuritis, glaucoma, and proliferative diabetic retinopathy. ${ }^{11-15}$. There is no definite evidence from this study that a lesion in this area is responsible for the contrast sensitivity abnormalities found in patients with WM.

The authors would like to thank Dr. L. Parapia, Consultant Haematologist, Bradford Royal Infirmary for allowing us to study patients under his care and Drs. T. Jenkins and W. Douthwaite for advice about the pattern ERG recording. We would also like to thank the patients studied for their patience and cooperation.

\section{References}

${ }^{1}$ Waldenstrom J: Incipient myelomatosis or 'essential' hyperglobulinaemia with fibrinogenopenia -a new syndrome? Acta Med Scand 1944, 117, 216-47.

2 Orellana J and Friedman AH: Ocular manifestations of multiple myeloma, Waldenstrom's macroglobulinaemia and benign monoclonal gammopathy. Surv Ophthalmol 1981, 26, 157-69.

${ }^{3}$ Rosen ES, Simmons AV and Warnes TW: Retinopathy of Waldenstrom's macroglobulinaemia: photographic assessment. Am J Ophthalmol 1968, 65: 696-706.

${ }^{4}$ Schwab PJ et al.: Reversal of retinopathy in Waldenstrom's macroglobulinaemia by plasmapharesis. Arch Ophthalmol 1960, 64: 515-21.

5 Thomas EL et al.: Irreversible visual loss in Waldenstrom's macroglobulinaemia. $\mathrm{Br} J$ Ophthalmol 1983, 67: 102-6.

${ }^{6}$ Dawson WW, Trick GL and Litzgow C: An improved electrode for electro-retinography. Invest Ophthalmol Vis Sci 1979, 18: 988-91.

${ }^{7}$ Friedman $\mathrm{AH}$ et al.: Immunofluorescent studies of the eye in Waldenstrom's macroglobulinaemia. Arch Ophthalmol 1980, 98: 743-6.

${ }^{8}$ Davies-Jones GAB, Preston FE and Timperley WR: Neurological complications in clinical haematology, Blackwell 1980.

${ }^{9}$ Arden GB: The importance of measuring contrast sensitivity in cases of visual disturbance. $\mathrm{Br} \mathrm{J}$ Ophthalmol 1978, 62: 198-209.

${ }^{10}$ Bodis-Wollner, I: Vulnerability of spatial frequency channels in cerebral lesions. Nature 1976, 261: 309-11.

${ }^{11}$ Maffei L and Fiorentini A: Electroretinographic responses to alternating gratings before and after section of the optic nerve. Sceince 1981, 211: 953-5.

12 Fiorentini A et al.: The ERG in response to alternating gratings in patients with diseases of the peripheral visual pathway. Invest Ophthalmol Vis Sci 1981, 21: 490-3.

${ }^{13}$ Bobak $\mathrm{P}$ et al.: Pattern electroretinograms and visual evoked potentials in glaucoma and multiple sclerosis. Am J Ophthalmol 1983,96: 72-83.

14 Wanger P and Persson HE: Pattern-reversal electroretinograms in unilateral glaucoma. Invest Ophthalmol Vis Sci 1983, 24: 749-53.

${ }^{15}$ Arden GB et al.: Pattern electroretinograms become abnormal when background diabetic retinopathy deteriorates to a preproliferative stage. Br J Ophthalmol 1986, 70: 330-5. 